\title{
“WE HAVE ALWAYS BEEN THERE...”: DE FEMINISMOS AFRODIASPÓRICOS Y LA PRODUCCIÓN DE EULALIA BERNARD LITTLE ${ }^{1}$
}

\author{
MARIANELA MUÑOZ-MUÑOZ \\ Universidad de Costa Rica
}

\begin{abstract}
Mientras el aparato crítico sobre la escritora afrocostarricense Eulalia Bernard Little afirma su foco en la lucha negra, sugiere la neutralidad o ausencia de las discusiones de género, o incluso, una sobreatención a la figura masculina en su poesía. Sin embargo, hasta el momento no ha habido una revisión que permita valorar el tratamiento de las experiencias simultáneas de raza y género en el transcurso de su carrera literaria. Mediante una revisión diacrónica del conjunto de sus publicaciones y activismo poético, este artículo examina el lugar fundamental ocupado por el ser y la visión de las mujeres negras y su relación con una tradición de pensamiento y movilización de los feminismos afrodiaspóricos. La centralidad de su propio cuerpo y la referencia a la maternidad de herencia africana y caribeña pueden rastrearse desde los primeros trabajos de Bernard; pero, además, la autora otorga un mayor protagonismo a la imagen y vínculos con figuras femeninas, escritoras, divinidades de la diáspora africana y activistas, de manera progresiva, en sus últimos poemarios. La coincidencia entre estos gestos, las producciones de otras autoras afrodescendientes, los movimientos feministas afrolatinoamericanos y una epistemología derivada de la experiencia de las mujeres negras, nos permite iniciar una conversación sobre las contribuciones de Bernard Little a la génesis de un pensamiento afrofeminista y de la diáspora en Costa Rica. Al mismo tiempo, pone en evidencia las complejidades del uso y (auto) adscripción de los feminismos negros, sus antecedentes y la diversidad de sus experiencias en el contexto del Caribe y Centroamérica.
\end{abstract}

PALABRAS CLAVE: raza y género, pensamiento afrofeminista, literatura afrocostarricense, diáspora africana, maternidad negra.

"We have always been there...": On Afro-diasporic Feminisms and Eulalia Bernard Little's Writing

While critics of Afro-Costa Rican writer Eulalia Bernard Little affirm her focus on the Black struggle, they suggest neutrality or absence of gender discussions, even excessive attention to the male figures in her poetry. However, to date there has not been a review that assesses the treatment of the simultaneous experiences of race and gender in her literary career. Through a diachronic review of her publications and her poetic activism, this article examines the

\footnotetext{
${ }^{1}$ Este artículo es un resultado del proyecto "Pensamiento político de Eulalia Bernard Little: poeta, activista y docente universitaria" inscrito en la Vicerrectoría de Investigación de la Universidad de Costa Rica. El trabajo cuenta, además, con el auspicio del programa "Connected Worlds: The Caribbean, Origin of the Modern World", financiado por el European Union's Horizon 2020research and innovation programme (Marie Skłodowska-Curie grant agreement $n .^{\circ} 823846$ ).
}

Muñoz-Muñoz, Marianela (2021), “'We have always been there...': de feminismos afrodiaspóricos y la producción de Eulalia Bernard Little", Lectora, 27: 145-173. ISSN: 1136-5781 D.O.I.: 10.1344/Lectora2021.27.7, marianela.munoz@ucr.ac.cr

Recepció: 15 de desembre de 2020 - Acceptació: 21 de juny de 2021 
fundamental space occupied by the being and vision of Black women throughout her oeuvre and its relationship with the Afro-diasporic feminist tradition of thought and mobilization. The centrality of her own body and the reference to motherhood of African and Caribbean heritage can be traced back to Bernard's early works; however, the author increases the attention to the image of and links with female figures, writers, deities of the diaspora and activists in her latest collection of poems. The coincidence between these gestures, the work of other Afro-descendant female authors, the Afro-Latin American feminist movements, and an epistemology derived from the experience of Black women, allows us to initiate a conversation about Bernard Little's contributions to the genesis of a Black feminist and diaspora thought in Costa Rica. At the same time, the analysis highlights the complexities of the use of and (self) ascription to afro-feminisms, its antecedents, and the diversity of its experiences in the Caribbean and the Central American context.

KEY WORDS: race and gender, Black feminist thought, Afro-Costa Rican literature, African diaspora, Black motherhood.

Para Pamela Cunningham (y sus preguntas sobre Eulalia)

More voices in recent days have emerged within the black community present in the metropolitan, predominantly mestizo area, to voice the rights and values of the afrocostarican people. Not surprising at all, these are female voices, young black, women voices. -Eulalia Bernard LitTle, Ciénaga $=($ marsh $)(2006)$

En agosto de 2019, la Cátedra de Estudios de África y el Caribe de la Universidad de Costa Rica organizó el "Primer Encuentro de mujeres escritoras afrodescendientes". En este marco, e incluso en homenajes anteriores, la producción multilingüe de Eulalia Bernard Little (Puerto Limón, 1935-2021) y su contribución cultural y política a la comunidad afrocostarricense y a la diáspora, en general, ocuparon un lugar de privilegio. ${ }^{2}$ Su trabajo fue celebrado como vehículo para la afirmación de la identidad afrocaribeña; particularmente, desde una lúcida y lúdica conciencia crítica sobre las dinámicas sociales y económicas costarricenses. Sin embargo, luego de una mesa dedicada al abordaje de la figura masculina y el erotismo en la escritura de Eulalia Bernard, se produjo una calurosa discusión sobre los límites o alcances de su discurso de género. ¿Cómo conciliar una aparente reverencia al hombre negro y cierta visión conservadora sobre la mujer con la Eulalia disruptiva y transgresora? Mientras el epígrafe nos revela cómo, para Bernard, "not surprising at all”, son las voces de mujeres jóvenes quienes se manifiestan por los

\footnotetext{
${ }^{2}$ La Cátedra de Estudios de África y el Caribe de la Universidad de Costa Rica organizó un homenaje a la vida y obra de Eulalia Bernard el 12 de noviembre del 2018.
}

146

Lectora, 27 (2021): 145-173. ISSN: 1136-5781 D.O.I.: 10.1344/Lectora2021.27.7 
derechos y valores del pueblo afrocostarricense, una nueva generación de afrofeministas interrogó la interrelación de raza y género en la precursora de la literatura de autoras negras del país. ${ }^{3}$ Sin demandar una figura monolítica ni soslayar su humanidad maravillosamente contradictoria, varias participantes del encuentro expresaron su inquietud por conocer cómo Eulalia Bernard se nutría de y era capaz de motivar las luchas políticas y culturales de sus congéneres en la actualidad.

El abordaje o ausencia de las discusiones de género y su subordinación a la temática racial, o bien la misma centralidad de la figura masculina en el conjunto poético de Bernard, han sido anteriormente abordados por la crítica $y$, como se mencionó, han provocado cierta polémica. Sin embargo, hasta el momento no se ha realizado una revisión diacrónica que examine el tratamiento del sujeto femenino y sus transformaciones a lo largo de su carrera literaria. Una lectura que considere, además, las discusiones sobre las producciones de escritoras afrodescendientes de las Américas y el Caribe y hasta qué punto una epistemología derivada de la experiencia de las mujeres negras — según el abordaje de diferentes teorías sobre los feminismos de color- aporta nuevos horizontes de análisis. A su vez, nos permite iniciar una conversación sobre las contribuciones de Eulalia Bernard a la génesis de un pensamiento afrofeminista y de la diáspora negra en Costa Rica.

En este marco, y tomando en cuenta la interrelación entre la escritura y el activismo cultural de Eulalia Bernard Little, nuestro artículo propugna el lugar fundamental ocupado por el ser y la visión sobre la mujer negra en el conjunto de su escritura poética. Se plantea que, conforme afianza su carrera literaria, Bernard otorga un mayor protagonismo a esta imagen y a los vínculos con sus congéneres, incluyendo escritoras, divinidades y activistas de la diáspora. Esto parece coincidir con los movimientos y políticas de identidad de mujeres afrolatinoamericanas que se cristalizan durante la década de los noventa y con los cuales la autora se relaciona (Rosario Fernández, 2015: 359).

El análisis considera su producción como parte de una "compleja, amplia y heterogénea perspectiva de pensamiento que surge de las experiencias de vida cotidianas de las mujeres africanas, negras y afrodescendientes en el mundo entero", según la definición de feminismos afrodiaspóricos propuesta por Figueroa y Hurtado (2014: 113). Identificamos la utilidad de este término relacionado con los feminismos negros como una vía productiva para el abordaje de la contribución intelectual y artística de Bernard por su localización en contextos latinoamericanos y la posible inclusión de quienes consideran "que sus luchas no son una forma de

\footnotetext{
${ }^{3}$ La herencia colonialista subyace en la elaboración de las categorías raciales y la distinción entre blancos y negros. Pese a su carácter de constructo, el artículo incorpora la terminología negro (a), afrocaribeño (a), afrocostarricense y afrodescendiente para referir de manera indistinta a las identidades racializadas, culturales y políticas de estas poblaciones.
} 
feminismo, o que no desean llamarlo como tal, pero cuyos esfuerzos buscan la equidad de género y étnico-racial, adicionales a las propuestas de vinculación de los hombres [...] como aliados" (Figueroa y Hurtado, 2014: 113). ${ }^{4}$

Cabe aclarar que el empleo de tal terminología no asume una identidad de Eulalia Bernard como feminista, pues la misma autora siempre rechazó llamarse así; inclusive y pese a que la designación "womanist" — de origen afroamericanoencontró un mayor eco entre las activistas e intelectuales afrocaribeñas, Bernard tampoco acogió esta identificación. ${ }^{5}$ Independientemente del nominativo, el uso de cualquiera de estas categorías analíticas respeta una diferencia entre las prácticas políticas de las mujeres o bien sus propios procesos de "aceptación (o rechazo) de la palabra feminismo". Esta salvedad, planteada por Morris en su trabajo con mujeres creole de la Costa Caribe de Nicaragua, informa nuestra visión, pues "de este modo, podemos reconocer cómo las personas definen su propia identidad y no imponerles identidades específicas, al mismo tiempo que mostramos cómo su política se cruza con movimientos políticos más amplios y se nutre de ellos" (2010: 236-237).

Tomando en cuenta estas advertencias, en la segunda sección exponemos la discusión de género - o su ausencia - en el quehacer y producción de Eulalia Bernard según lo ha planteado el aparato crítico. Nuestra lectura arguye la viabilidad de una lectura afrofeminista, tanto en virtud de la centralidad - erótica- del cuerpo en sus escritos y activismo (Lorde, 2007) como de la transformación de su posicionamiento sobre las relaciones entre género y raza a través del tiempo. Como evidencia del carácter dinámico de la propuesta de Bernard, los apartados subsiguientes examinan cómo, en el conjunto de su escritura, la autora

\footnotetext{
${ }^{4} \mathrm{Al}$ respecto, resulta esclarecedora la observación de la intelectual afrofeminista brasileña Sueli Carneiro, citada por Curiel: "Desde este punto de vista se podría decir que un feminismo negro, construido en el contexto de sociedades multirraciales, pluriculturales y racistas - como son las sociedades latinoamericanas - tiene como principal eje articulador al racismo y su impacto sobre las relaciones de género dado que él determina la propia jerarquía de género de nuestras sociedades" (2002: 103).

${ }^{5}$ La reflexión de la filósofa e intelectual jamaicana Sylvia Wynter al referirse al trabajo de escritoras afrocaribeñas da cuenta de las tensiones con las mismas terminologías. En el epílogo "Beyond Miranda's Meanings: Un/silencing the 'Demonic Ground' of Caliban's 'Woman'” del tomo Out of the Kumbla: Caribbean Women and Literature, Wynter señala cómo el término "wo manist", tomado de la feminista afroamericana Alice Walker, revela una serie de contradicciones asociadas con los mismos marcos de referencia de las mujeres - escritoras y críticas - afroamericanas y caribeñas. Añade la necesidad de otro término para describir estas experiencias y lecturas; en sus palabras: "we shall need to translate the variable 'race', which now functions as the intra-feminist marker of difference, impelling the dually 'gender/beyond gender' readings" (Boyce Davies y Fido, 1990: 364).
} 
afrocostarricense participa de tradiciones, gestos y discursos políticos sobre la mujer negra que se repiten a la largo de la diáspora africana. La tercera sección identifica la centralidad de la figura materna y la politización de la maternidad negra en el proceso de autoidentificación, creación artística y compromiso social de la(s) autora(s) afrodescendiente(s) (Boyce Davies, 1994; Collins, 2000; Walker, 2004). Bernard expone en su poesía las particularidades de las experiencias de las mujeres negras mediante la misma visión de su madre y su papel en la comunidad (diaspórica) afrocaribeña. De manera complementaria, el cuarto apartado recupera una intensificación de vínculos con sus congéneres, artistas y activistas políticas e, inclusive, con divinidades femeninas de origen africano (Valdés, 2014) en el transcurso de su producción textual. Bernard forja un listado (Cooper, 2017) - homenaje- de los nombres y contribuciones de mujeres y espiritualidades negras; en este conjunto, el activismo afrofeminista de organizaciones a nivel local y regional está presente también.

En virtud de una progresiva recuperación y politización de las identidades de las mujeres negras, la producción cultural de Eulalia Bernard se enmarca en una tradición de feminismos afrodiaspóricos y es germen de un pensamiento afrofeminista en Costa Rica. Ahora bien, tal revalorización de sus textos y activismo cultural no pretende negar la coexistencia (y contradicciones) de un discurso radical y transgresor de mujer negra con otros, de corte "conservador", sino más bien evidenciar el dinamismo de su posicionamiento como intelectual a lo largo de su carrera. Figuras como Eulalia Bernard Little complican las discusiones sobre la relación entre raza y género, apuntan a sus antecedentes y variaciones a lo largo de la diáspora y, al mismo tiempo, lo sitúan en el contexto del Caribe y Centroamérica.

\section{Navegando las aguas de los feminismos afrodiaspóricos}

Las posibles lecturas "feministas" de la figura de Eulalia Bernard han desafiado a quienes se acercan al conjunto de su obra escrita. Como en el caso de otras autoras centroamericanas que inician su producción cerca de la década de los setenta y ochenta, Bernard se resiste a ciertas etiquetas. Desde la historiografía costarricense, Bernard puede considerarse como una de las escritoras de la ruptura e, inclusive, como parte del movimiento contracultural en el cual se inscriben las autoras centroamericanas de su época. Eulalia inaugura su carrera poética en un contexto en el cual, según Zavala, los temas "adquieren contenidos eminentemente políticos" y donde "nuevos sujetos sociales femeninos escriben literatura en la región, esto es, se diversifica el origen social y étnico de las escritoras. También se amplían y diversifican las tendencias estéticas representadas" (2011: 59-60). En este sentido, la efervescencia política de los setenta influye en su tratamiento de la lucha de clases; aunque, en su caso, la crítica al statu quo se verifica desde una identidad racial y étnica-lingüística diferenciada (Ravasio, 2020a). Cada una de 
estas consignas - raza, clase y diferencia cultural - parecen superponerse a las discusiones de género en el conjunto de una producción que desafía los mitos de homogeneidad hispánica y blanquitud costarricense. ${ }^{6}$

En su condición de escritora de la diáspora africana y caribeña, el proyecto estético-político de Eulalia Bernard ha sido enmarcado en movimientos de carácter transnacional a favor de la lucha negra (Ravasio, 2020b; Gallego, 2012; Meza, Zavala y Toledo, 2015). Desde sus primeras incursiones en el escenario cultural, Bernard se posiciona como una abanderada de la Negritud, el movimiento poético y político convocado por los caribeños Aimé Césaire y Léon Damas y el senegalés Léopold Sédar Senghor en las primeras décadas del siglo XX (Césaire, 1995); pero también como heredera del Garveyismo, que influye directamente en las dinámicas de activismo de su comunidad en la provincia de Limón (Harpelle, 2002; Putnam, 2013). La autora celebra el legado del panafricanismo, las conferencias y festivales negros inaugurados por intelectuales y políticos como W. E. B. Dubois, Blaise Diagne y Kwame Nkrumah (Drake, 1982). Bernard Little se asocia así con una trayectoria de movilización en donde la condición particular de opresión de las mujeres negras y sus contribuciones para la liberación racial no ocupan una atención particular (Sharpley-Whiting, 2002; Leeds, 2013; Nardal, 2014; Blain, Leeds y Taylor, 2016; McDuffie, 2016). En palabras de Boyce Davies, y como en el mismo caso de la Negritud: "certain versions of African Nationalism, Pan-Africanism and Afrocentrism become discourses which turn on the concept of a unicentricity and imply the exclusion or subordination of women's issues or questions of sexual identity or difference within" (1994: 7-8).

Pese a lo anterior y al igual que otras "mujeres de color" que la precedieron, las formas mediante las cuales Eulalia Bernard participa de y encarna estos proyectos transgrede la neutralidad - masculina - de sus mismos andamiajes políticos. Al respecto, cabe resaltar la experiencia del Primer Congreso de la Cultura Negra de las Américas, realizado en agosto de 1977 en Cali, Colombia, en donde Ratcliff recupera los nombres y el protagonismo de una serie de escritores afrolatinoamericanos y figuras intelectuales. Junto a Eulalia Bernard, menciona a Manuel Zapata Olivella y Jorge Artel (Colombia); Nelson Estupiñán Bass (Ecuador),

\footnotetext{
${ }^{6}$ Los discursos fundacionales costarricenses celebran la homogeneidad cultural y racial —blanquitud - de sus pobladores como características que diferencian la emergente nación del resto de naciones centroamericanas (Putnam, 1999; Molina, 2002). Las mismas mitologías blancas decimonónicas y su perpetuación resultan confrontadas por generaciones de descendientes jamaicanos en Costa Rica, desde sus procesos de reclamo de derechos de ciudadanía (Senior Angulo, 2011) hasta los esfuerzos por el reconocimiento de las contribuciones de la comunidad afrocaribeña al desarrollo del país y del mismo carácter multicultural de la nación (Muñoz-Muñoz, 2017).
} 
Abdias do Nascimento, Olivia Avellar Serna y Eduardo de Oliveira (Brasil); Gerardo Maloney y Carlos Guillermo Wilson (Panamá) (2008: 27). Los nombres de las dos mujeres, Bernard y Avellar, reaparecen luego en la conformación del comité ejecutivo del evento, en donde Manuel Zapata Olivella funge como presidente, el investigador afropanameño Roy Simón Bryce-Laporte como secretario y como delegados ejecutivos Eulalia Bernard, Olivia Sera Avellar y Wande Abimbola (32). El protagonismo de Eulalia Bernard, en medio de una intelectualidad negra y masculina, parece alinearse con el carácter progresista del encuentro. Más aún, las discusiones sobre los desafíos derivados de la imbricación de género y raza en la experiencia de las mujeres afrodescendientes ocupan un espacio en los registros y recomendaciones de uno de los grupos de trabajo del mismo congreso.

La incursión de la escritora afrocostarricense en espacios (masculinos) de discusión sobre justicia racial parece ser un hecho recurrente en su trayectoria político-cultural. Así consta en las actas del Primer Seminario sobre la situación del negro en Costa Rica en 1978. La secuencia de hombres del comité organizador aparece interrumpida por el único nombre de mujer: Eulalia Bernard Little. La misma voluntad de negociación y creación de espacios para la participación en la esfera pública y la generación de políticas a favor de la comunidad afrocostarricense, que motiva el desarrollo del seminario, es encarnada por Bernard en su trayectoria de vida. "Eulalia abrió espacios, abrió el camino", en palabras de la exdiputada Joycelyn Sawyers (2016: s.p.). Así lo considera también la poeta afrocostarricense Shirley Campbell Barr, quien se refiere al impacto del activismo y escritura de Bernard dentro y fuera de Costa Rica en los siguientes términos:

Hay un antes y un después de Eulalia Bernard [...]. Ella es un hito en la historia de los afrocostarricenses y a ella le debemos un homenaje de verdad. Porque Eulalia tomó la decisión de visibilizar algo que no existía, en la persona de ella. Eso fue importante, para todos nosotros, los que venimos detrás de Eulalia. Eulalia como escritora, como activista, como mujer negra, que tomó la decisión de hacer algo [...] siempre con la bandera de que ella es una mujer negra y eso yo creo que es lo más importante [...]. Yo voy afuera de Costa Rica, mire, y que en Costa Rica somos tan poquitos, pero yo voy afuera de Costa Rica, recientemente y no sólo recientemente, porque me ha pasado otras veces [...]. Estaba en la Universidad de North Carolina, en Chapel Hill y [...] una escritora jamaicana que estaba ahí,

\footnotetext{
${ }^{7}$ Para Ratcliff, "By acknowledging the double burden of gender and race faced by Afrodescendant women as well as the need to support women's liberation, this resolution is indicative of the progressive political tendencies and centrality of women at el Congreso and in the nascent movements for Afro-descendent and Pan-African solidarity" (2008: 34).
} 
me dice: - ¿qué se hizo esta señora, Eulalia, que estuvo en no sé dónde? -Ah, Eulalia ahí está, bien. En Brasil, me preguntaron varias veces: - ¿Y Eulalia? Esta poeta costarricense... A Eulalia la conocen en muchas partes, porque ella decidió hacer una historia. Decidió escribir una historia nueva para los negros de Costa Rica, [...] por eso, nosotros le debemos mucho a Eulalia, le debemos mucho y hay que darle honor. (2016: s.p.; el énfasis es nuestro)

Eulalia Bernard irrumpe "con la bandera de que ella es una mujer negra" en una agenda local y transnacional a favor de las poblaciones afrodescendientes; una estrategia que repite "en la persona de ella", en su producción poética. Smart (1987), uno de los primeros investigadores de la literatura afrocentroamericana, subraya la fortaleza y liderazgo de la mujer negra como características del proyecto de liberación de Bernard, tanto en el quehacer público como en su primera publicación impresa. No obstante, y anticipando el abordaje de la crítica sobre el tratamiento del tema de género, el crítico señala que las preocupaciones sociales en Ritmohéroe (1982) "are not gender-based, but group- and race-based". Más aún, concluye que el posicionamiento de Bernard "appears to betray no gender-based conflict. Both as a woman and as an artist she is harmoniously integrated into her group" (84).

La idea de una integración "armoniosa" que diluye cualquier discusión de género en el espacio textual se repite en trabajos posteriores. Sharman (2000) sugiere inclusive que la referencia a favor de la mujer en algunos poemas, como en el caso de “To Women's Liberation”, “does not necessarily call for a reorganization of gender roles, rather it seeks the valorization of 'women's roles' among Limonenses" (75). Desde su lectura, el mismo poema podría relativizar el movimiento por la diferencia e inclusive la necesidad de una "liberación" específica para las mujeres, en la medida que la hablante lírica enfatiza una relación armoniosa de complementariedad donde, según los versos iniciales y conclusivos del poema: "The Problem is not the Difference / But the Interference... / This is the way I see it" (Bernard, 1991: 14).

Ahora bien, dado que en este poema la "interferencia", utilizada con una clara función de paralelismo fonético con "diferencia", no llega a aclararse en el resto de la exposición, nuestro análisis plantea que no es posible afirmar si se trata de una crítica a lo que se ha denominado feminismos de la igualdad, o bien de la diferencia (Curiel, 2002: 100). Cuanto sí parece inferirse es una expectativa de complementariedad, horizontal y placentera, la cual se repite en el conjunto de la producción poética de la afrocostarricense. Así, en las siguientes estrofas de “To Women's Liberation", se recuperan una serie de imágenes explicativas sobre la convivencia placentera entre lo (entendido como) masculino y femenino: la abeja y la flor, la 
luna y el sol, niños y niñas que siendo pequeños disfrutan, desnudos, construyendo un castillo de arena, o quienes al crecer juegan por el cambio de sus voces: "Discovering the pleasure / Of not being alike, and yet; / The miracle of their coexistence" (Bernard, 1991: 14).

En la misma tónica de relativización de una lucha específica a favor de la mujer, Sharman indica que Bernard incluye a ambos, hombres y mujeres, en su denuncia de las inequidades estructurales. Más aún, apunta que "Bernard's valorization of men in her poetry is a conscious part of her desire to rehabilitate a local Limonense sense of self, a cohesive identity for the city and the region" (2000: 76). Por un lado, considera que la afirmación del "hombre" por parte de la poeta consigue abarcar a las mujeres, como parte de un colectivo "limonense"; por otro, sugiere cierta actitud soteriológica de la autora en relación con la figura masculina. Específicamente, en la publicación donde la autora incluye el debatido y polisémico “To Woman's Liberation”, My Black King (1991), Sharman identifica una función de rehabilitación para el hombre negro. Agrega — de manera debatibleque éste lo requiere por haber padecido los peores estragos de la esclavitud y el racismo.

Precisamente, el esmero en la tematización de la figura masculina por parte de Eulalia Bernard, sobre todo en el poemario My Black King, ha llamado la atención de la crítica especializada y, como se mencionó en la introducción de este artículo, su tono de reverencia desconcierta a nuevas generaciones de lectores y lectoras. La estudiosa de la literatura afrocostarricense, Dorothy Mosby, se refiere a algunos de los textos como una celebración de la masculinidad negra que incluye, además, el reconocimiento de "male historical personages such as Marcus Garvey, Martin Luther King Jr., and Louis Armstrong” (2003: 77). En la medida que muchos de los poemas de My Black King (1991) constituyen la versión escrita de las primeras declamaciones del vinilo Negritud (1976), se comprende que el gesto de revisión histórica que recupera figuras políticas y artísticas visibles de la diáspora no se restringe a la publicación impresa (Muñoz-Muñoz, 2020). No obstante, llama la atención la centralidad del hombre afrodescendiente que Bernard procura desde la dedicatoria de My Black King, "to all the men of my family... [who] have given me a beautiful childhood, challenging adolescence and creative adulthood"; añade, inclusive, un reconocimiento a su amante, "specially" Marco Antonio, quien reaparece como interlocutor o destinatario del yo poético en varios de los versos de la colección.

En el recurso paratextual de la dedicatoria y en el contenido de diversos poemarios, McKinney (1996) identifica una ruptura con la imagen "ausente o negativa" del hombre negro en la escritura de otras mujeres "particularmente del 
Caribe". ${ }^{8}$ Desde su análisis, la reivindicación de esta figura cobra particular relevancia en el poema "Dock Workers", donde la mirada pasa del cuerpo físico al símbolo político y se transforma "into an evocation of their multiple roles in the making of both Costa Rican and world history: 'Boxing champions; football heroes, / Carnival dancers.., rioters; / Loaders of ships, lovers of sex, / Port of hopes..." (1996: 15). El poema, junto a otros de My Black King (1991), son considerados por Senior-Grant como "un tributo al hombre negro, tributo que a la vez comparte con otros" (2019: 12). Mediante el título del poemario y el texto de igual nombre, Bernard "corona al hombre negro siguiendo la vieja tradición de los pueblos ancestrales tales como los Ashanti. Está llena de orgullo ante este hombre investido de poder y extasiada ante el cuerpo que brilla cada mañana con un sudor dulce y que la induce a ofrecerse a él" (12; el énfasis es nuestro).

Si bien diferimos de algunas de las conclusiones de la crítica en relación con la sobreatención de la figura masculina, las primeras producciones de Bernard - particularmente, My Black King - parecen apuntar hacia una ausencia o relativización de un discurso a favor de la mujer negra. Por un lado, el foco imaginado neutral, pero masculino, de la lucha negra, parece superponerse a una crítica específica sobre las relaciones entre raza y género por parte de la autora; con ello, Bernard se distanciaría de un discurso feminista negro por el riesgo de "dividir la lucha negra", una de las acusaciones padecidas por el movimiento y desmentidas por el colectivo Combahee River y otras intelectuales feministas afroamericanas ya desde la década de los setenta (Moraga y Anzaldúa, 2015).

Por otro lado, aun cuando la reverencia a la figura masculina "investida de poder" a la cual se "ofrece" la poeta sugiere cierta relación jerárquica entre los roles de género, la celebración del cuerpo y ser del hombre negro va de la mano de la manifestación del erotismo de un yo-mujer-negra. ${ }^{9}$ Es decir, en la conexión y comunicación del deseo interior se verificaría el gesto afrofeminista de lo erótico; según, Audre Lorde, "an assertion of the lifeforce of women; of that creative energy empowered, the knowledge and use of which we are now reclaiming in our language, our history, our dancing, our loving, our work, our lives” (2007: 55). En conexión con lo erótico, y tal cual lo plantea la poeta y crítica afroamericana, la mujer negra se afirma y rechaza "those other supplied states of being which are not

\footnotetext{
${ }^{8}$ Gallego plantea una observación semejante sobre el poema "Yo tampoco" (200), donde Bernard "speaks back to a demeaning and univocal definition of black men as hyperagressive and violent" (2012: 85).

${ }^{9}$ La autora agradece al crítico Franklin Perry el recordatorio sobre un trabajo de análisis pendiente acerca del erotismo en la poesía de Eulalia Bernard. Una revisión en profundidad sobre este tema se visualiza como complemento de nuestro argumento.
}

154

Lectora, 27 (2021): 145-173. ISSN: 1136-5781 D.O.I.: 10.1344/Lectora2021.27.7 
native to me (her), such as resignation, despair, self-effacement, depression, self -denial" (58).

Junto al enunciado del deseo erótico, la misma inclusión del cuerpo de Eulalia Bernard en el proyecto poético sugiere una forma de activismo textual, entendido como un proceso en el cual las mujeres de color se hacen presentes, corporalmente, en los textos que escriben y hablan (Cooper, 2017: 3). En el proceso de revisión de la obra escrita de la poeta afrocostarricense es posible identificar una ruptura del estereotipo del cuerpo femenino pasivo y su transformación en un símbolo poético y político y un(a) agente de cambio (McKinney, 1996: 15). Ya sea mediante el uso de la primera persona o las apelaciones y diálogos con personajes históricos, Bernard propone la centralidad de su voz y emociones de mujer negra para relacionarse intelectual, sensorial y afectivamente con diversas personalidades y producciones artísticas de la diáspora. Si bien muchos de estos referentes son masculinos, ella se afianza como una interlocutora autorizada e informada que desafía las jerarquías de género impuestas y forja un diálogo horizontal y emancipatorio $(\mathrm{Mu}-$ ñoz-Muñoz, 2020).

La activista política presente en espacios imaginados como masculinos, la escritora que parece neutralizar la lucha de género en favor de la raza, la poeta devota del hombre negro y la mujer que afirma su yo deseante, complica, de muchas formas, el uso de y la (auto)adscripción a los feminismos negros. Sin embargo, es precisamente la transformación y acentuación del tema de género en el transcurso de su carrera literaria lo que nos permite afirmar que la producción de Eulalia Bernard navega las aguas del feminismo afrodiaspórico. Como se mencionó, Figueroa y Hurtado definen este tipo de feminismo "como una compleja, contradictoria, amplia y heterogénea perspectiva de pensamiento, de acción política y de vida, que emerge de las realidades que protagonizan las mujeres afrodescendientes en diferentes momentos de la historia y espacios geográficos" (2014: 114). Se valora cómo en las formas textuales emerge un pensamiento feminista negro, como un "punto de vista diferente, aunque heterogéneo", que deriva de las experiencias individuales y comunitarias del ser mujeres negras" (Collins, 2000: 5). Ampliando el archivo intelectual, Collins incluye "formas narrativas, testimoniales, tradiciones orales y literarias (incluidas la poesía y la prosa)", además de otras observaciones etnográficas para la identificación de una epistemología feminista negra (275).

En esta dirección, a lo largo de su carrera literaria y particularmente en su madurez, Eulalia Bernard otorga y reclama un lugar para los nombres, cuerpos, espíritus, lecciones de mujeres afrodescendientes y sus luchas. Como lo anticipa Mosby, Bernard "places the female voice at the center of her poetry in roles as savior, agent, and lover, as well as the voice of social and cultural consciousness" (2007: 175). Al igual que otras escritoras de la diáspora, se trata entonces de una práctica encarnada de activismo que puede incluir la evocación de la madre y su 
influjo en el proceso de autoidentificación, creación artística y compromiso social. De igual forma y en consonancia con una práctica de alianzas entre mujeres negras, Bernard celebra los vínculos con sus congéneres, artistas y activistas políticas, con divinidades femeninas de origen africano e inclusive con organizaciones afrofeministas. Cada una de estas líneas temáticas y su coincidencia con una tradición de pensamiento y activismo de los feminismos afrodiaspóricos se analizan en los siguientes apartados.

\section{Madre y maternidad política negra en la poesía de Bernard}

Desde el primer producto cultural de la autora, el poemario discográfico Negritud (1976), Bernard asegura un lugar de privilegio para la madre negra. En la secuencia de los seis actos de su vinilo, específicamente entre los actos 3 y 4, la performer comparte el intermedio o "Special" para declamar su poema "Intangible Love". Los versos son precedidos por un fragmento de "Love's Theme" de la orquesta (de mujeres) del cantante afroamericano Barry White e incluyen una dedicatoria: "to all mothers, especially to my mother and all Black mothers of the world" (Bernard, 1976). ${ }^{10}$ En el proyecto estético-político inaugural de la autora, la celebración de la maternidad negra requiere un acto único. El poema, que emula un diálogo entre madre y su hijo(a), quien le pide pruebas de amor ("Kiss-kiss"), permite una revisión del estereotipo de la fuerte matriarca, identificado por Patricia Hill Collins como una de las imágenes de control de la experiencia de las mujeres negras (2000: 174). De manera lúdica, Bernard ilustra cómo el ser maternal excede las muestras superfluas de amor o caricias de la "dulce madre". A diferencia de la mestiza identificada por su apellido hispánico "Miss Ramírez”, la madre negra y caribeña - quien prepara "tea" y "bami" y utiliza "Castor oil or sorosi tea" cuando el niño está enfermo- asegura el bienestar y progreso de los suyos mediante un amor intangible, según se ilustra en las últimas estrofas:

... Mama; what do you?

Make you so busy

You no give me kiss-kiss

Like Miss Ramirez give

Her pickney them every minute.

\footnotetext{
${ }^{10}$ Sobre las particularidades del producto inaugural Negritud de Eulalia Bernard, en términos de performance poética, ver Ravasio (2020b) y Muñoz-Muñoz (2020). Negritud (1976) es un producto que alterna música y declamación y así ocurre con este poema-acto único que luego será incluido con variaciones mínimas en el poemario My Black King (1991), siempre dentro de un acto denominado "Special".
}

156

Lectora, 27 (2021): 145-173. ISSN: 1136-5781 D.O.I.: 10.1344/Lectora2021.27.7 
... So a that a bother you... ah!

Well..., here, kiss, kiss, kiss.

A not going sew your new dress,

Nor work fi pay your school fee,

No cook you ackee and codfish.

A just going sit down...,

And kiss, kiss, kiss, to ris.

... Mama; what do you?

A have no new dress

Fi recite in Sunday School.

A have no pencil, fi me homework.

Oh!... Lord..., me belly feel funny

and all you do is just look pan me,

and a kiss kiss me to ris.

That no love man;

That a "Poppy Show"...

... Well son I'm glad you find out se (that)

That Black mother's love is intangible.

(Bernard, 1976: acto “Special”)"11

Ahora bien, junto a lo que pueda sugerir un rol tradicional de la mujer negra en el hogar, por el cuidado familiar, Bernard recupera un detalle de la organización social y cultural caribeña en los versos: "Nor work fi pay your school fee". La frase sintetiza la condición trabajadora del grupo femenino y la importancia histórica de la educación para las familias afro-costarricenses (Rosario Fernández, 2015: 265). Mediante este guiño, la celebración de la madre negra se acerca no sólo a las de su comunidad sino, y tal cual lo indica en su dedicatoria, a quien la engendrara: Carolina Bernard. De esta forma, la labor y el legado de la maestra de inglés Carolina, su preocupación por la educación de la infancia negra y sus lecciones de vida, se entretejen afectivamente en la memoria y creación de la hija escritora y activista. La última rememora en su última frase y palabra cuando declama "con suavidad

\footnotetext{
${ }^{11}$ Los fragmentos corresponden a una transcripción de lectura poética incluida en el acetato $\mathrm{Ne}$ gritud (1976) y la citación de los versos se indica con el acto (sección) del disco donde se ubica la declamación. Ver Ravasio (2020b) para un análisis de las variaciones y el alcance de la propuesta oral y escrita de "Intangible Love" incluyendo el guiño diaspórico del uso de la mayúscula para Black Mother.
} 
y lentitud, es decir, con tempo pausado y articulación lenta y abierta para darle mayor delicadeza amorosa al cierre del diálogo" (Ravasio, 2020b: 367).

Bernard mantendrá el tono autorreferencial y evocará a su madre en varios textos a lo largo de su carrera (Lago Graña, 2007); particularmente, mediante los posesivos "mi" o "my" según el idioma del poema, junto al nominativo "madre". Como ejemplo emblemático, se encuentra el poema "Mi madre y el tajamar" que la autora añade en la versión escrita del referido acto intermedio "Special", en la publicación My Black King (1991). ${ }^{12}$ De carácter autobiográfico, las estrofas inician ubicando al yo lírico ante un paisaje marítimo que conecta el Caribe con el llamado Atlántico Negro y, en última instancia, con el continente originario, África. En primera persona, la hablante enuncia cómo por la "lengua materna" y "de los labios de ella", la madre, aprende "la solemne verdad / sobre mis antepasados / (En esta tierra abandonados)". Hay una vinculación entre el aquí, marcado por el "esta tierra", con el allá de una historia anterior, la de los "abuelos koromanti / Tus abuelos fanti”. La madre, transmisora de memoria ancestral, incide en la construcción de una identidad política, pues gracias a ella aprende también "a ser soldado / con la palabra desenvainada / en la mano" (28). El "lenguaje entrecortado" de la madre, que sugiere los desafíos del bilingüismo de la comunidad afrocaribeña, precede el momento de la contemplación / reflexión sobre el paisaje e historia heredada y los hace cómplices con "una mirada de orgullo lejano" (29).

El peso de la figura materna en el proceso de construcción de la identidad personal resulta fundamental y coincide con las narrativas de identidad del Caribe negro. Al respecto, Chamberlain (2006) reconoce un fundamento epistemológico diferenciado en la forma de presentarse de los sujetos, quienes mediante la mención de sus genealogías de línea materna manifiestan un particular sentido del ser (9). En el caso de la producción literaria de Bernard, la identificación de un legado afectivo y una conciencia política por parte de su madre negra coincide además con la experiencia de otras escritoras de la diáspora. Las figuras de la madre, abuelas y mujeres anteriores en el linaje familiar permiten la construcción de una memoria ancestral que incide en el presente de las autoras y define su identidad como mujeres negras (Hampton, 1995). En palabras de O’Reilly, y en relación con la producción de Toni Morrison, "Black female subjectivity generally, and creativity specifically, are formed, nurtured, and sustained through women's identification with, and connection to, their mother line (O'Reilly, 2004: 15). ${ }^{13}$

\footnotetext{
${ }^{12}$ Los versos de "Mi madre y el tajamar" resultan unos de los más conocidos y referidos de Eulalia Bernard. Ver la producción de la Casa Presidencial de Costa Rica en <www.youtube.com/ watch?v=SXlzxvU522s $>$.

${ }^{13}$ En su análisis sobre una teoría de la maternidad en la autora afroamericana Toni Morrison, O’Reilly afirma la recurrencia de una tradición matrilineal en la literatura de mujeres negras. La
} 
La evocación de la madre negra permite identificar una experiencia, subjetividad y discurso diferenciados. En el caso de las escritoras negras y caribeñas, un discurso "defined in opposition to the terms of motherhood accorded to white women, and with a different set of historical realities" (Boyce Davies, 1994: 136). Ello incluye, por ejemplo, la revisión de la imagen del "ángel del hogar" predominante en la poesía escrita por mujeres en la región; las relaciones jerárquicas entre los géneros y, en última instancia, el cuestionamiento de las divisiones y significados de los espacios público y privado para las mujeres negras a lo largo de la diáspora (Collins, 2000: 47).

En esta dirección, Eulalia Bernard articula una imagen específica de mujer desde la figura de su madre negra y su "única" (y diferenciada) voz. Por ejemplo, en otro poema del vinilo Negritud, la misma voz sobresale en una especie de panteón de sonidos "universales" (y de origen masculino) que son capaces de elevar el alma (negra). Luego de escuchar al violinista catalán Pablo Casals (primera estrofa), al líder afroamericano Martin Luther King (segunda estrofa), los sonidos desde África hacia América que se cristalizan en la trompeta de Louis Amstrong (tercera estrofa), la hablante lírica celebra como "Unique sounds": ${ }^{14}$

Numberless choires and choruses

in Latin, Greek, English and Spanish,

ringing for God the bells of their voices

and yet...

there was this unique voice among them,

They say it was my mother's.

(Bernard, 1976: acto 1)

Al respecto, Senior-Grant destaca cómo "la voz única de la madre se superpone a todas las otras de los célebres varones (Pablo Casals, Martin Luther King Jr. y Louis Armstrong)" e inclusive cómo "por encima de todos los coros se destaca la voz universal de la mujer-madre” (2019: 6). La misma voz-lengua es maestra en el poema "Mi madre y el tajamar" ya mencionado. De manera recurrente, la (su) madre se identifica entonces influyendo la formación de la conciencia y visión hacia "afuera" de la poeta. Por ejemplo, en el poema "Proyección" del acto 6 de

madre aparece como mentora y modelo que traslada su poder a su hija (2004: 14). La conexión intergeneracional, la hermandad y la comunicación de un pasado por parte de las figuras ancestrales son apuntadas a su vez por Hampton (1995) en su análisis de la poesía de la estadounidense Rita Dove y la costarricense Shirley Campbell. Sobre la última escritora, Ravasio ha señalado también la centralidad de la triada negra mujer-madre (2020a: 130).

${ }^{14}$ La versión escrita de este poema se recupera en la publicación My Black King (1991). 
Negritud, ${ }^{15}$ la hablante lírica se ubica en el exterior (techo) de su casa (materna) y ante la pregunta “¿Qué hice yo cuando niña?”, el yo responde en una suerte de monólogo y a la vez presagio: "Subir al techo de mi casa, / jugar de predicadora, / di tal discurso que... / mi madre del susto aplaudió". La mujer parece influir positivamente en la memoria y forja de su carácter de tal forma que "Invocarte"(la), como ocurre en uno de sus últimos poemarios: "es deletrear el éxito / es bailar con el mar / mientras tú (madre) ingenias sueños / que hacen brillar el tiempo" (Bernard, 2006: 35). Es decir, la madre negra facilita la ensoñación, materia fundamental de la carrera de Bernard, como lo ha subrayado antes Alice Walker sobre su propia experiencia creativa en su clásico texto-manifiesto In Search of Our Mothers' Gardens (1983).

En el caso de Eulalia Bernard, el ingeniar sueños conduce, además, a la acción. Puede incluso afirmarse que su conciencia de clase y compromiso político continúan un linaje de herencia maternal. Desde una visión afrodiaspórica, esta dinámica relacional comulga con una práctica de la institución de la maternidad negra que sirve como catálisis para el activismo social, tal y como lo ha señalado Patricia Hill Collins en relación con un pensamiento político negro y feminista (2000). Madres, "otras-madres" y "redes de mujeres" comparten una visión extendida de familia y un compromiso de cuidado con su comunidad; junto a ello, comprenden su rol "as a symbol of power and the activist mothering it might engender as an enduring theme that politicizes Black women" (2000: 194).

La contribución de la maestra Carolina es reconocida por Bernard y por su grupo desde la visión de una maternidad política. Además, "Mamá" transmite su activismo a su "linda niña", como se puede observar en el poema "Los negros", escrito en 1999 y publicado en 2006. Según este texto, el reconocimiento del lugar ocupado por los hombres trabajadores negros - es decir, "los negros negros"- se posibilita desde una visión de justicia intergeneracional, compartida entre mujeres:
Mamá me enseñó a conocer
a los negros negros
me llevaba al muelle a ver trabajar a
los negros negros
los ayudó a querer a
sus hijos negros,
a educar
a sus hijos negros
entonces ellos le responden

\footnotetext{
${ }^{15}$ La versión escrita de este poema aparece en Ritmo héroe (1996).
} 
con un gesto de reverencia

y le dicen

que linda su niña doña Carolina.

¿No es de revolucionarios

querer a sus hijos?...

(Bernard, 2006: 65)

Como en otros poemas, incluyendo el mencionado en el segundo apartado, “Dock Workers", Eulalia Bernard celebra en "Los negros" las contribuciones de una figura excluida del imaginario nacional. La hablante lírica dignifica la imagen del muellero porque su madre le "enseñó a conocer[lo]". En este sentido, llama la atención cómo tal conocimiento y la misma acción política a favor del grupo derivan de su madre. Inclusive, y como lo mencionara antes en su panfleto de presentación como candidata a diputada por el Partido Pueblo Unido para la contienda electoral de 1986, Eulalia Bernard se posiciona visibilizando una lucha emprendida por mujeres negras para la afirmación de su comunidad: "I have spoken out frankly about the extraordinary struggle of our women in the quest for identity and the preservation of our best traditions" (Bernard, 1986: 2).

Desde esta visión politizada de la maternidad negra y de las relaciones entre mujeres asociadas con esta experiencia-visión colectiva de cuidado comunitario, es posible comprender el porqué de la (gradual) mención de sus nombres a lo largo de la producción poética de Bernard. La relectura histórica de la vivencia del ser afrodescendiente, su carácter reivindicativo y el mismo germen de su conciencia política se desarrolla gracias al vínculo figurativo o activo con otras mujeres negras, como se analizará en la siguiente sección.

\section{Vínculos con mujeres, espiritualidades y activistas negras: un listado progresivo}

En otro trabajo, hemos señalado la relación entre movimientos y estéticas negras y la forja del pensamiento político de Eulalia Bernard. Más aún, identificamos el diálogo con intelectuales y figuras históricas o artísticas afroamericanas, caribeñas y africanas como uno de sus principales recursos discursivos (Muñoz-Muñoz, 2020). En esta sección, nos detendremos en el análisis de una serie de poemas que dan cuenta de los vínculos afrocéntricos y diaspóricos del yo poético con otras escritoras, artistas y activistas negras e inclusive divinidades. La revisión pretende evidenciar cómo desde los inicios de su carrera como escritora, e inclusive en su debatido poemario My Black King (1991), Bernard recupera el nombre o imagen de mujeres negras y subraya su lugar en la historia. Este recurso de mención 
o referencia se verifica de manera progresiva y coincide con la propuesta de Cooper en relación con la elaboración de "listados" como una estrategia que visibiliza la contribución de las mujeres negras. Para la estudiosa del pensamiento de mujeres afroamericanas, "these lists situate Black women within a long lineage of prior women who have done similar kinds of work, and naming those women grants intellectual, political, and/or cultural legitimacy to the Black woman speaking their names" (Cooper, 2017: 26).

El listado de Eulalia Bernard incluye los nombres de figuras históricas, legendarias; también los de otras escritoras afrodescendientes y sus trabajos. Además, en sus últimas producciones, la autora convoca a organizaciones de mujeres negras, tanto de carácter local, como internacional y cuyo activismo es abiertamente afrofeminista. Aun cuando algunas de sus menciones se intercalan con las de figuras masculinas, Bernard nombra, revalorizando, a otras mujeres con quienes se relaciona o identifica de diferentes formas. La acentuación de estas imágenes en el devenir de su carrera sugiere la construcción de un yo poético que oscila entre la recuperación de una memoria ancestral y la visión de transformación revolucionaria para el nuevo milenio. Si en la forja de una identidad y discurso político prevalece la imagen del líder negro, la poeta desestabiliza tal orden con la anotación y apropiación de algún referente femenino.

En esta dirección, a contrapelo de los discursos oficiales y colonialistas, en el poema "Emprendí un largo viaje", del acto 2, "Africa Soul", del poemario discográfico Negritud (1976), la autora narra un recorrido de (auto)conocimiento que empieza por la piel y el cuerpo, pero que luego pasa por la memoria oral de las hazañas de figuras emblemáticas, una de ellas una mujer negra. Precedida por la entonación de "Angelitos negros", a cargo de la afroamericana Roberta Flack, la voz de Bernard irrumpe y anuncia: ${ }^{16}$

Emprendí un largo viaje, descubrí que debajo de la piel, de los músculos, de los huesos, existe un océano.

\footnotetext{
${ }^{16}$ La afroamericana Roberta Flack, la cantante emblemática del "Killing me Softly" (1973) "se hace presente en su interpretación de 'Angelitos Negros' en la mayoría de los poemas del acto 2; en el acto 6, reaparece con su canción-protesta 'Compared to What' (1967); además, es protagonista de uno de sus poemas eróticos” (Muñoz-Muñoz, 2020: 22).
}

162

Lectora, 27 (2021): 145-173. ISSN: 1136-5781 D.O.I.: 10.1344/Lectora2021.27.7 
Escuché las leyendas de Harriet Tubman,

del lúcido Garvey,

del valeroso Chaka [...].

(Bernard, 1976: acto 2)

Las dos primeras estrofas de este poema inauguran un viaje iniciático que, conforme avanzan los versos, el yo concibe con valor futuro: "Viajaré eternamente". Como punto de partida, la hablante lírica descubre su piel (negra) bajo la cual existe el océano, metáfora de carácter metafísico, o bien una referencia al llamado Atlántico negro (Gilroy, 1993); en la medida en que, en la siguiente estrofa, el continente americano se conecta con el Caribe y éste con África a partir de la secuencia de las figuras Harriet Tubman (Estados Unidos), Marcus Garvey (Jamaica y el llamado Circuncaribe) y Chaka - Shaka Zulú- (Sudáfrica). La elección de tales referentes como un paradigma de conocimiento y revelación de la propia historia (negra) resulta peculiar; sobre todo, la figura inaugural del itinerario, cuya cruzada de liberación de centenares de personas esclavizadas en el Sur de los Estados Unidos la convirtió en el (la) "Moisés negro(a)". De los tres personajes, el femenino tiene nombre y apellido, mientras los masculinos, acentuando el ritmo del poema, se acompañan de sendos adjetivos. Asociar sus acciones con leyendas no resulta negativo, sino modélico y hasta profético; más aún, quienes se han acercado a la biografía de la propia Tubman reconocen cómo la forja personal de un mito sobre su accionar decanta en una causa "feminista" (Hobson, 2014: 4). ${ }^{17}$

Bernard repite este gesto del listado - reconocimiento- de mujeres negras en el transcurso de su producción. Así, en Ciénaga (2006), se incluye un poema de 1998 donde la hablante lírica comparte una especie de recapitulación de su experiencia con la Negritud y sus referentes. La autora reflexiona sobre su vínculo con el movimiento en términos eróticos, pues ha "degustado la Negritud / tiene sabor a cacao"; pero este placer/poder ha sido posible gracias a conversaciones "con King” (Martin Luther King), "Dubois” (W. E. B. Dubois) y “Toni, mi amiga” (Bernard, 2006: 60). Mientras las primeras relaciones son figuradas y distantes en el tiempo, el yo evidencia una cercanía con la escritora afroamericana Toni Morrison, no sólo por el apelativo "amiga", sino por llamarla por su nombre.

Tal gesto afectivo de la mención del nombre de otras autoras negras se repite en el mismo poemario y de manera casi antológica, en el texto "We fear not your

\footnotetext{
${ }^{17}$ Es interesante la valoración positiva de una leyenda sobre Tubman y su coincidencia con el análisis de esta figura por parte de Hobson. En sus palabras, "in crafting a narrative emphasizing her role as an Underground Railroad conductor, Tubman validated the struggle for women's rights [...] if she, a woman, could lead a successful battle during the Civil War, then surely women deserved the right to vote and the rights to full citizenship" (Hobson, 2014: 4).
} 
Gods". Junto a las figuras masculinas que han soñado a favor de la justicia negra: Garvey, King y Mandela, la hablante lírica enuncia la labor de las escritoras, pensadoras de la diáspora, con quienes se identifica por el uso de la palabra:

[...] We can put in writing the best thoughts to enforce heroic actions, as many of our sisters do:

Maya Angelou, Toni Morrison, Merle Collins, Miriam Alves,

Nancy Morejon, Carol Boyce, Opal Palmer, my

Compatriots

Shirley Campbell and Delia McDonald.

Our everlasting, Louise Bennet. All exquisite

Voices of our African queens.

As Mirriam Da Costa puts it "Daughters of the

Diaspora”.

(Bernard, 2006: 119)

La secuencia cruza el continente americano (Estados Unidos y Brasil) y el Caribe (Cuba y Jamaica) hasta encontrarse con la producción nacional y con quien fuese su modelo lingüístico y político de performance poética, Louise Bennet. ${ }^{18}$ En primera persona del plural, la autora celebra un colectivo creativo negro y femenino y evidencia el saber literario que alcanza la labor de la crítica especializada, según la alusión de Carol Boyce y de la investigadora DeCosta-Willis y su libro editado Daughters of the Diaspora: Afra-Hispanic Writers (2003), en donde aparece el trabajo de Bernard. Esta relación afectiva y de fuentes se repite en el poema "Mandela" de Tatuaje (2011) mediante la recuperación del nombre de dos de las poetas antes mencionadas. En los primeros versos y, antes de asumir, gozar o inmortalizar lo negro "a lo Mandela", la hablante lírica enuncia un deseo de proyección: "Yo / quiero a esa negra / rotundamente bella / a lo Shirley / con tristeza erguida a lo Maya" (Bernard, 2011: 46). Simultáneamente, Bernard demuestra la cercanía y validación de dos creadoras y acaso sus poemas más conocidos y politizados: la afrocostarricense Shirley Campbell y su "Rotundamente negra" (Mosby, 2016: 33) y la afroamericana Maya Angelou con su "Still I Rise" (Boyce Davies, 1994: 151).

\footnotetext{
${ }^{18}$ Sobre la función poético-política de las performances de la llamada Miss Lou, ver Edmondson (2009). Un análisis de la influencia de Louise Bennett en Eulalia Bernard se reconoce como una tarea pendiente.
}

164

Lectora, 27 (2021): 145-173. ISSN: 1136-5781 D.O.I.: 10.1344/Lectora2021.27.7 
El mapa de relaciones para la generación de conocimiento de mujeres negras (Cooper, 2017: 31) se extiende hasta África mediante figuras artísticas y -como en el caso de Tubman-histórico-legendarias. Volviendo a Negritud (1976), y en el acto 5, "Freedom", Bernard elige la canción "Pata pata" de la sudafricana Miriam Makeba (1932-2008) como su complemento musical. El hecho es significativo cuando se considera la indisolubilidad de las carreras artística y política de Makeba, desde su campaña contra el apartheid de su país hasta su vínculo con las Panteras Negras en Estados Unidos. Posteriormente, en uno de los poemas que recibe el premio Griot en 1996, la poeta afrocostarricense se apropia de la imagen de un modelo femenino de resistencia contra el poder colonial y esclavista durante el siglo XVII en el territorio hoy conocido como Angola. ${ }^{19}$ Tal como se verifica en diversas prácticas espirituales y culturales de la diáspora (Butler, 1998), Bernard se iguala con la reina heroica Nzingha, divinizándola(se), ennegreciéndola(se) y replicándola(se) en otras mujeres - princesas - africanas por el empleo de la primera persona:

\author{
Soy la Diosa Nzingha \\ Mis vulvas están repletas \\ de negrísimas semillas $[\ldots]$ \\ Soy la Diosa Nzingha \\ Inicio mis conjuros \\ ataviada de negro intenso \\ y negro carmesí.
}

Cómo resplandezco en las encías

de princesas africanas $[\ldots]$.

(Bernard, 2006: 41)

La escritora afrocostarricense expande sus geografías afectivas y estético-políticas mediante la relación asertiva con otras figuras y espiritualidades de origen africano ya desde My Black King, donde imágenes de deidades "from ancient days" cuelgan de sus orejas y la hacen sentir auténtica, según el poema "Authentic"

\footnotetext{
${ }^{19}$ Según una entrevista recuperada por Shirley Jackson, Eulalia Bernard es la primera mujer afrodescendiente que gana un premio Griot en Latinoamérica. El "griot" constituye una figura de la tradición oral africana que narra historias para la preservación de la memoria cultural y política de su comunidad. Bernard la define en los siguientes términos "We griots are preachers who express the power of words"; añade, además, su relación con Nzingha y África: "Remember Nzingha? My history goes way back to Africa. I am an Akan” (Jackson, 2003: 123-124).
} 
(Bernard, 1991: 42). En la misma colección, Bernard dedica unos versos a la "Obeah woman" porque "She can do wonder / The Obeah woman yonder / She can lift you up / And she can bring you down..." (18). Revisando los prejuicios de una práctica espiritual asociada con "la magia negra" en el Circuncaribe (Putnam, 2013: 50), la autora le otorga género y un poder sobrenatural que relativiza los límites entre el bien y el mal: "Whatever you beg for son"; en última instancia, desenmascara la ingenuidad de quien la busca para conocer su futuro: "Obeah woman, je jé! / Only know the past" (Bernard, 1991: 18). El poemario evidencia así un diálogo con otros sistemas religiosos - no eurocéntricos- e inclusive con una tradición epistémica africana.

Complementariamente, al repetir el recurso estético-político de evocación de divinidades de la espiritualidad negra, Eulalia Bernard coincide con otras escritoras a lo largo de la diáspora. Según Valdés (2014), la presencia de entidades y religiones traídas desde el oeste de África - particularmente yorubas - en la producción de diversas autoras afrodescendientes a lo largo de América y del Caribe sugieren una búsqueda de modelos alternativos de "ser mujer", "one that celebrates both body and mind, both sacred and secular" (Valdés, 2014: 18). Identificadas como hijas de Ochún ("Oshun's daughters"), poetas y narradoras se asemejan a sus orishas y a la diosa Yemanyá como seres "that are beautiful, wealthy, fierce, sexual, and all warriors" (11). No parece fortuito, entonces, encontrar la recuperación de estas figuras por Bernard y su consecuente afirmación de un yo femenino negro. La afrocostarricense continúa con esta tradición y reclama con autoridad "Háblame tierra / así piden / su hogar / mis Orishas y dioses" en el poema "Háblame" (Bernard, 2011: 29). Además, concibe una visión de trascendencia y heroísmo para la mujer — también experiencia erótica para la pareja—; un "conjuro" que se verifica gracias a las divinidades africanas quienes "tienen la palabra", según la conclusión de su poema tripartito "El siglo XXI se llama mujer":

La hermosa reina del mar

Matsu inicia sus conjuros de amor

con su pueblo.

Yemayá dueña de

las aguas fluye en el

alma del varón y lo llena

de placer.

Una vez más con júbilo

Isis y Osiris

se unen en segundos

contra los oráculos.

Las diosas tienen la Palabra.

166

Lectora, 27 (2021): 145-173. ISSN: 1136-5781 D.O.I.: 10.1344/Lectora2021.27.7 
Mujer tómala, y vuela sin temor.

(Bernard, 2006: 80)

El poema resulta significativo desde su título hasta su carácter progresivo. Asumiendo una función profética, en la primera parte del poema, la hablante lírica declara el nombre del nuevo siglo "mujer" y designa lo que "es": "persona", "madre", "amiga" "que hace / ríe, llora, escribe / estudia, viaja, trabaja" (acaso una definición forjada desde sí misma). Los versos añaden desde una perspectiva interseccional que articula la raza, el género, la clase y la sexualidad, cuanto "ya no será": "esclava”, "violada”, "pobre” (Bernard, 2006: 77). El oráculo, in crescendo, apunta a una nueva experiencia de liberación que pasa por el conocimiento, la autodefinición de lo "propio", su tópico recurrente sobre "la belleza del equilibrio", para alcanzar la comunicación y apropiación de las fuerzas ancestrales de las diosas. Se trata de una visión esperanzadora y transgresora, aun con los resabios que pueden considerarse conservadores en la construcción del género.

Eulalia Bernard dedica "El siglo XXI se llama mujer" al Centro de Mujeres Afrocostarricenses, organización con una clara agenda afrofeminista de lucha contra la discriminación racial y de género. ${ }^{20}$ Las fundadoras del Centro son, además, protagonistas en la conformación de la Red de Mujeres Afrocaribeñas y Afrolatinoamericanas, en cuya primera reunión en Santo Domingo, República Dominicana, Eulalia Bernard encabeza la delegación de Costa Rica junto a Epsy Campbell (Rosario Fernández, 2015: 359). A cinco años de la creación de la Red, Bernard escribe y les dedica el poema que sirve de título a este artículo. Reconociendo un colectivo de mujeres heterogéneas en donde algunas "argumentan que sus luchas no son una forma de feminismo, o que no desean llamarlo como tal, pero cuyos esfuerzos buscan la equidad de género y étnico-racial" (Figueroa y Hurtado, 2014: 113) - según la definición de feminismos afrodiaspóricos- la hablante lírica corona su visión política de las mujeres negras y acaso de sí misma afirmando:

We have always been there.

We are the miracle of survival.

We have taken care of lands of people,

We have dug up foolishness

We have warred disguised poverty.

\footnotetext{
${ }^{20}$ La autora agradece a la Dra. Paola Ravasio la observación sobre estas dedicatorias y el manejo (político) de los paratextos por parte de Eulalia Bernard.
} 
We are indomitable $[\ldots]$

Yes, you are queens, warriors, higglers,

Teachers, prostitutes and preachers.

Yes, you are daughters of Isis [...]

I have the infula to infuse justice.

(Bernard, 2006: 85)

En un recorrido desde la experiencia ancestral, de resistencia esclavista, de lucha por la incorporación social, Bernard caracteriza a la mujer negra como indómita. Reconoce además su múltiple ser, ampliando los roles ya mencionados en sus poemas “To Women's Liberation" o hasta "El siglo XXI se llama mujer”. Esta vez, las mujeres afrodescendientes (de la Red) son reinas, guerreras, "higglers" - figura jamaicana que rescata la labor de cultoras y vendedoras de los productos de la tierra-, maestras, prostitutas, predicadoras. Como "salida al atrapamiento producto de la inclusión/exclusión en las narrativas dominantes” (Meza, Zavala y Toledo, 2015: 128), se presentan diversas y desafiantes de una sociedad racista, clasista y patriarcal. En última instancia, la visión de justicia, encarnada por la autora en la madurez de su producción como una ínfula, celebra la constante existencia y lucha de la mujer negra, quien "siempre ha estado allí...".

\section{A modo de conclusión}

Las producciones culturales de Eulalia Bernard navegan una tradición de feminismos afrodiaspóricos que recupera y, a la vez, politiza las identidades de las mujeres negras. Desde su experiencia situada de afrocaribeña en Costa Rica, Bernard lleva su cuerpo, sus deseos y su propia visión de transformación social a los diferentes espacios de discusión sobre los destinos de la comunidad y a su literatura. Lo que a primera vista sugiere una desatención de la cuestión del género en sus textos merece ser reconsiderado desde una mirada diacrónica que identifique la persistencia y transformación del ser y la visión sobre la mujer negra. Desde sus primeras obras y, con una mayor acentuación en sus últimos trabajos, Eulalia Bernard concibe un lugar para los nombres, cuerpos, espíritus, lecciones de mujeres afrodescendientes y sus luchas; ello incluye el tópico de la maternidad política y la elaboración de listados que honran el legado y visión de futuro de quienes comparten un "punto de vista diferente, aunque heterogéneo" en virtud de la coincidencia de las dinámicas de raza y género. Ambas estrategias afirman la condición de las mujeres negras y de sus contribuciones históricas y, a la vez, de sí misma.

La carrera literaria de Bernard desestabiliza el racismo, pero también las expectativas de subordinación a estructuras patriarcales. La afirmación erótica de la

168

Lectora, 27 (2021): 145-173. ISSN: 1136-5781 D.O.I.: 10.1344/Lectora2021.27.7 
hablante lírica, característica del conjunto de su producción, apunta hacia la construcción de relaciones horizontales y coloca a su cuerpo de mujer negra como espacio de experiencia, conocimiento y activismo. La visibilización del protagonismo de la imagen de y relaciones con otras figuras femeninas de la diáspora, en el plano afectivo, político-cultural y espiritual, se intensifica, además, en el transcurso de su carrera; precisamente, en consonancia con su relación con los movimientos y políticas de identidad de mujeres afrolatinoamericanas que se cristalizan durante la década de los noventa.

Independientemente del empleo de tal término por parte de las intelectuales o activistas y en el caso de la misma Eulalia Bernard, un análisis conjunto de su producción escrita y quehacer político-cultural sugiere la génesis de un pensamiento político afrofeminista en Costa Rica (y Centroamérica). La circulación de una epistemología de mujeres negras se hace evidente mediante imágenes y temas que coinciden con los de otras autoras afrodescendientes. Ahora bien, en este diálogo con otras autoras a lo largo de las Américas y el Caribe y en el encuentro con las nuevas generaciones de escritoras y activistas comprometidas con la justicia social, racial y de género en Costa Rica, Bernard aporta el dinamismo de su escritura encarnada, sus disonancias por la coexistencia (y contradicciones) de un discurso radical y transgresor de mujer negra con otros, de corte "conservador"; pero, ante todo, la constancia y acentuación de su(s) presencia(s).

\section{REFERENCIAS BIBLIOGRÁFICAS}

Bernard, Eulalia (1976), Negritud, San José CR, INDICA. [vinilo]

-(1986), "Candidate to Congress for the United People Party for February 1986

'Hold the Stars'”, propaganda política de la autora para la contienda de 1986.

Limón, Costa Rica: 1-4. [sin publicar]

—(1991), My Black King: A Collection of Poetry, Eugene OR, World Peace

University.

-(1996), Ritmohéroe, San José CR, Editorial Costa Rica.

-(2006), Ciénaga = (marsh), San José CR, Ediciones Guayacán.

-(2011), Tatuaje, San José CR, Ediciones Guayacán.

Blain, Keisha N.; Asia Leeds y Ula Y. Taylor (2016), "Women, Gender Politics, and Pan-Africanism”, Women, Gender, and Families of Color, 4 (2): 139-145.

Boyce Davies, Carole (1994), Black Women, Writing, and Identity: Migrations of the Subject, Londres \& Nueva York, Routledge.

Boyce Davies, Carole and Elayne Savory Fido (eds.) (1990), Out of the Kumbla: Caribbean Women and Literature, Trenton NJ, Africa World Press. 
Butler, Kim D. (1998), "Ginga Baiana - The Politics of Race, Class, Culture, and Power in Salvador, Bahia”, Afro-Brazilian Culture and Politics: Bahia, 1790s to 1990s, Hendrik Kraay (ed.), Nueva York \& Londres, M. E. Sharpe: 158-176.

Campbell, Shirley (2016), Comunicación personal, Alajuela, Costa Rica, entrevistada por Marianela Muñoz-Muñoz. Inédita.

Césaire, Aimé (1995), "What Is Negritude to Me?", African Presence in the Americas, C. Moore; T. R. Saunders y S. Moore (eds.), Trenton NJ, Africa World Press: 13-19.

Chamberlain, Mary (2006), Family Love in the Diaspora, Londres, New Transaction Publishers.

Collins, Patricia Hill (2000), Black Feminist Thought: Knowledge, Consciousness, and the Politics of Empowerment, Nueva York, Routledge.

Cooper, Brittney C. (2017), Beyond Respectability: The Intellectual Thought of Race Women, Urbana IL, University of Illinois Press.

Curiel, Ochy (2002), "Identidades esencialistas o construcción de identidades políticas: El dilema de las feministas negras", Otras miradas, 2: 96-113.

DeCosta-Willis, Miriam (ed.) (2003), Daughters of the Diaspora: Afra-Hispanic Writers, Kingston, Ian Randle Publishers.

Drake, St Clair (1982), "Diaspora Studies and Pan-Africanism”, Global Dimensions of the African Diaspora, Joseph E. Harris (ed.), Washington, D. C., Howard UP: 341-402.

Edmondson, Belinda (2009), Caribbean Middlebrow: Leisure Culture and the Middle Class, Ithaca NY, Cornell UP.

Figueroa, Aurora Vergara y Katherine Arboleda Hurtado (2014), "Feminismo afrodiaspórico. Una agenda emergente del feminismo negro en Colombia”, Universitas humanística, 78: 109-134.

Gallego, Mar (2012), “On Both Sides of the Atlantic: Hybrid Identity and the Spanish-Speaking Diaspora in Agnès Agboton, Mónica Carrillo and Eulalia Bernard", Migration, Narration, Identity: Cross-Cultural Perspectives, Peter Leese; Carly McLaughlin y Władysław Witalisz (eds.), Fráncfort, Peter Lang: 73-90.

Gilroy, Paul (1993), The Black Atlantic: Modernity and Double Consciousness, Cambridge MA, Harvard UP.

Hampton, Janet Jones (1995), "Portraits of a Diasporan People: The Poetry of Shirley Campbell and Rita Dove", Afro-Hispanic Review, 14: 33-39.

Harpelle, Ronald (2002), The West Indians of Costa Rica: Race, Class, and the Integration of an Ethnic Minority, Montreal \& Londres, McGill-Queen's UP.

Hobson, Janell (2014), “Harriet Tubman”, Meridians, 12 (2): 1-8.

170

Lectora, 27 (2021): 145-173. ISSN: 1136-5781 D.O.I.: 10.1344/Lectora2021.27.7 
Jackson, Shirley (2003), “'Our Weapon is a Strong Language’: A Conversation with Eulalia Bernard", Daughters of the Diaspora: Afra-Hispanic Writers, Miriam DeCosta-Willis (ed.), Kingston, Ian Randle Publishers: 122-128.

Lago Graña, Josefa (2007), “Identidad afro-caribeña en Costa Rica: La poesía de Eulalia Bernard", Con-Textos, 19 (39): 69-84.

Leeds, Asia (2013), “Toward the 'Higher Type of Womanhood': The Gendered Contours of Garveyism and the Making of Redemptive Geographies in Costa Rica, 1922-1941", Palimpsest: A Journal on Women, Gender, and the Black International, 2 (1): 1-27.

Lorde, Audre (2007), Sister Outsider: Essays and Speeches, Berkeley CA, Crossing Press.

McDuffie, Erik (2016), “The Diasporic Journeys of Louise Little: Grassroots Garveyism, the Midwest, and Community Feminism", Women, Gender, and Families of Color, 4 (2): 146-170.

McKinney, Kitzie (1996), "Costa Rica's Black Body: The Politics and Poetics of Difference in Eulalia Bernard's Poetry", Afro-Hispanic Review, 15 (2): 11-20.

Meza, Consuelo; Magda Zavala y Aida Toledo (comp.) (2015), Mujeres en las literaturas indígenas y afrodescendientes en América Central, Aguascalientes, Universidad Autónoma de Aguascalientes.

Molina, Iván (2002), Costarricense por dicha. Identidad nacional y cambio cultural en Costa Rica durante los siglos xix y xx, San José CR, Editorial de la Universidad de Costa Rica.

Moraga, Cherríe y Gloria Anzaldúa (eds.) (2015), This Bridge Called My Back: Writings by Radical Women of Color, Albany, SUNY Press. [1981]

Morris, Courtney Desiree (2010), "Pensar en el feminismo afronicaragüense", Política e identidad. Afrodescendientes en México y América Central, Odile Hoffman (coord.), México, Centro de Estudios Mexicanos y Centroamericanos, 233-269.

Mosby, Dorothy (2003), Place, Language, and Identity in Afro-Costa Rican Literature, Columbia, University of Missouri Press.

-(2007), "Writing Home: Afro-Costa Rican Poets Negotiating Limón and San José", Unfolding the City: Women Write the City in Latin America, Anne Lambright y Elisabeth Guerrero (eds.), Columbia, University of Minnesota Press: $167-188$.

-(2016), “Traveling Words: A Reflection on 'Rotundamente negra' and AfroDescendant Women's Cultural Politics”, Meridians, 14 (2): 25-45. 
Muñoz-Muñoz, Marianela (2017), "Mujeres afrocostarricenses y multiculturalismo tardío: reforma de la constitución de la República (blanca) de Costa Rica”, América Latina Hoy, 77: 67-92.

-(2020), "Diálogos de Eulalia: la forja de una intelectual negra en Centroamérica", Poligramas, 51: 12-36.

Nardal, Paulette (2014), Beyond Negritude: Essays from Woman in the City, Albany, SUNY Press.

O'Reilly, Andrea (2004), Toni Morrison and Motherhood: A Politics of the Heart, Albany, SUNY Press.

Putnam, Lara (1999), "Ideología racial, práctica social y Estado liberal en Costa Rica", Revista de Historia, 39: 139-186.

-(2013), Radical Moves: Caribbean Migrants and the Politics of Race in the Jazz Age, Chapel Hill, University of North Carolina Press.

Ratcliff, Anthony (2008), “'Black Writers of the World, Unite!': Negotiating PanAfrican Politics of Cultural Struggle in Afro-Latin America", The Black Scholar, 37 (4): 27-38.

Ravasio, Paola (2020a), Black Costa Rica. Pluricentrical Belonging in Afra-Costa Rican Poetry, Würzburg, Würzburg UP.

-(2020b), "Negritud de Eulalia Bernard", Caribbean Worlds. Mundos Caribeños. Mondes Caribéens. Sammelband Forschungstage zur Karibik, Gabriele Knauern y Ineke Phaf-Rheinberger (eds.), Madrid \& Berlín, Iberoamericana/Verveurt: 347-372.

Rosario Fernández, Reina (2015), Identidades de la población de origen jamaiquino en el Caribe costarricense: segunda mitad del siglo XX, Santo Domingo, Cocolo Editorial.

Sawyers, Joycelyn (2016), Comunicación personal, Limón, Costa Rica, entrevistada por Marianela Muñoz-Muñoz. Inédita.

Senior Angulo, Diana (2011), Ciudadanía afrocostarricense: el gran escenario comprendido entre 1927 y 1963, San José CR, Editorial UNED.

Senior-Grant, Alder (2019), "Eulalia Bernard poeta de la Negritud costarricense y la imagen del Hombre Negro", ponencia presentada en el "Primer Encuentro de mujeres escritoras afrodescendientes”, Universidad de Costa Rica, San José, Costa Rica, 21-22 de agosto: 1-14. [sin publicar]

Sharman, Russell Leigh (2000), "Poetic Power: The Gendering of Literary Style in Puerto Limon", Afro-Hispanic Review, 19 (2): 70-79.

Sharpley-Whiting, T. Denean (2002), Negritude Women, Minnesota, University of Minnesota Press.

172

Lectora, 27 (2021): 145-173. ISSN: 1136-5781 D.O.I.: 10.1344/Lectora2021.27.7 
Smart, Ian (1987), "Eulalia Bernard: A Caribbean Woman Writer and the Dynamics of Liberation", Letras Femeninas, 13: 79-85.

Valdés, Vanessa (2014), Oshun's Daughters: The Search for Womanhood in the Americas, Albany, SUNY Press.

Walker, Alice (2004), In Search of Our Mothers' Gardens: Womanist Prose, Orlando FL, Houghton Mifflin Harcourt. [1983]

Wynter, Silvia (1990), “Beyond Miranda's Meanings: Un/silencing the 'Demonic Ground' of Caliban's 'Woman', Out of the Kumbla: Caribbean Women and Literature, Carole Boyce Davies y Elayne Savory Fido (eds.), Trenton NJ, Africa World Press: 355-372.

Zavala, Magda (2011), Con mano de mujer: antología de poetas centroamericanas contemporáneas (1970-2008), Heredia CR, Editorial Interartes. 